\title{
Études littéraires
}

\section{Gerda Zeltner, La Grande Aventure du roman français au $X X^{e}$ siècle, traduit de l'allemand par Christine Kubler, Paris, gonthier, 1967, 224 p.}

\section{Réal Ouellet}

Volume 1, numéro 2, août 1968

Roman et théâtre au XVIII ${ }^{\mathrm{e}}$ siècle

URI : https://id.erudit.org/iderudit/500033ar

DOI : https://doi.org/10.7202/500033ar

Aller au sommaire du numéro

\section{Éditeur(s)}

Département des littératures de l'Université Laval

ISSN

0014-214X (imprimé)

1708-9069 (numérique)

Découvrir la revue

Citer ce compte rendu

Ouellet, R. (1968). Compte rendu de [Gerda Zeltner, La Grande Aventure du roman français au $X X^{e}$ siècle, traduit de l'allemand par Christine Kubler, Paris, gonthier, 1967, 224 p.] Études littéraires, 1(2), 316-319.

https://doi.org/10.7202/500033ar d'utilisation que vous pouvez consulter en ligne.

https://apropos.erudit.org/fr/usagers/politique-dutilisation/ 
parler un « savant », ni même (il s'en défendait) un " intellectuel ».

L'auteur le sait qui parfois le rappelle. Comment peut-il alors nous parler du scientisme de Valéry (p. 145)?

Plus encore que l' « intellectualisme » qu'on lui prêtait, ce mot eût fait bondir le poète.

Valéry est un mystique: " $\mathrm{Ce}$ mysticisme sans objet qui est $\mathrm{MOI}$ » (Cahiers V, p. 806). Il suffit d'ouvrir les Cahiers pour s'en apercevoir. C'est pourquoi la troisième partie, la Mystique de l'arbre contient les meilleures pages - et les plus neuves - de cette thèse, dont la documentation sérieuse et riche est surtout extraite des Cahiers. Mais ici encore, bien des répétitions et des confusions. Or la Mystique de Valéry, bien qu'elle comporte comme toute mystique « des valeurs infinies ou absolues »- essentiellement irrationnelles - (Cahiers XIV, p. 750) n'est pas fondée sur le vague. Elle est d'abord exigence d'un esprit, qui, dans sa « folie de la clarté ", voudrait qu'en lui et par lui, - comme en Dieu qui est sa connaissance, - fût résolu « le conflit entre l'être et le connaître ". Cette formule, valéryenne par excellence, qui revient dans les Cahiers comme un leit-motiv, n'a pas échappé à l'auteur. II en saisit l'importance et lui consacre une page et demie, ce qui est trop peu, et malheureusement dans la seconde partie, où, envisagée surtout du point de vue philosophique, elle ne trouve pas toute sa résonance. En effet cet « asymptotisme de l'être et du connaître » (Cahiers, IX, p. 807) ressortit surtout, comme son " alpha » et son " oméga », à la Mystique valéryenne: elle explique son origine et son échec. « Folie est de suivre la ligne divine! » (Cahiers VIII, p. 501).

Quant à la Mystique de l'arbre, exemplaire de celle de Valéry, on la trouve sans doute dans cette troisième partie, mais encore émiettée à travers de minuscules chapitres, au nombre de six. Tout se tient pourtant dans cette Mystique. C'est cette cohérence que, dans l'axe de l'Arbre, il eût fallu montrer depuis le grand "Rêve de transcendance » qui engendre l'ArbreDieu, jusqu'à ses prolongements, amoureux et esthétiques, à travers l'Arbre-Amour et l'Arbre-Poème.

$$
*^{*} *
$$

Malgré ces imperfections surtout de construction - ce livre est important. Non seulement, par ce « mythe personnel » de l'arbre, il nous introduit au plus profond de Valéry, mais il répond au désir, souvent exprimé par le poète, en cela précurseur de Bachelard, de voir mieux étudier « less phénomènes de I'Imagerie mentale » (EFuvres, Pléiade, t. I, p. 1193). Car « c'est avec notre propre substance que nous imaginons, et que nous formons une pierre, une plante, un mouvement, un objet. Une image quelconque n'est peut-être que le commencement de notre âme » (ibid., p. 1233).

\section{Université Laval}

\section{Louis MORICE}

\section{Gerda ZELTNER, la Grande}

Aventure du roman français au $X^{\Theta}$ siècle, traduit de l'allemand par Christine Kubler, Paris, Gonthier, 1967, 224 p.

Depuis 1955, avec les innombrables discussions nées de la publication des premiers écrits théoriques d'Alain Robbe-Grillet, les études sur le roman français se sont multipliées. Mais les ouvrages d'ensemble importants de la critique française demeurent peu nombreux: on peut citer, entre autres, les livres de Maurice Nadeau, R.-M. Albérès et et Ludovic Janvier. Trop souvent, comme chez Jean-Bertrand Barrère (la Cure d'amaigrissement du roman, 1964) ou Kléber Hædens (Paradoxe 
sur le roman, nouvelle édition augmentée, 1964), les pirouettes verbales et les simplifications à outrance remplacent la discussion intelligente. La critique étrangère le livre de Gerda Zeltner une fois de plus nous incite à le croire comprend souvent mieux le nouveau visage de la littérature française que la critique française elle-même.

$\mathrm{M}^{\mathrm{me}}$ Zeltner se défend bien d'écrire une histoire du roman français de 1930 à nos jours; elle cherche plutôt « à fixer dans ses traits essentiels le visage que quelques auteurs, profondément conscients de l'insuffisance de la forme traditionnelle, ont donné au roman » (p. 8). Pour ce faire, elle tente d'abord de décrire le contexte historique qui a fait évoluer le roman traditionnel français voué le plus souvent à l'étude de la psychologie ou à la description de la " tranche de vie ". 1930 constitue une date charnière dans l'évolution de la société occidentale. Après l'explosion d'optimisme et la folle effervescence qui ont suivi la première guerre mondiale, on commence à se rendre compte, avec la crise économique américaine, la montée de l'hitlérisme et la transformation de la révolution soviétique que « les lendemains qui chantent » sont encore fort éloignés. Des livres aussi différents que $/ e$ Chiendent de Queneau, I'Imposture de Bernanos, Un barbare en Asie de Michaux, montrent, au dire de Maurice Nadeau, cité par Mme Zeltner, que «la confiance dans le progrès et l'ascension (naturelle > de l'humanité s'évanouit de même que la croyance au bonheur personnel procédant d'une maîtrise toujours plus assurée de l'homme sur les choses ». Notre époque est moins celle du progrès de la conscience de l'humanité que celle des révolutions, des révoltes, des guerres totales « avec leurs longues théories de déportés ou de réfugiés. Les grands mouvements de masse jouent un rôle de premier plan; le destin particulier, intercepté par la trajectoire des forces mécaniques, est projeté dans un univers qui, privé de tout ordre compréhensible, n'est plus ressenti comme cohérent. L'Histoire a quitté le domaine de l'individu, de l'homme en tant que participant aux destinées des peuples, pour s'intéresser au développement des masses ». Par ailleurs, avec l'essor de la psychanalyse, l'exploration du cosmos et la recherche atomique, " toutes les frontières ont disparu, à l'intérieur comme à l'extérieur »" (pp. 12-16).

Le roman contemporain devait ressentir les contrecoups de telles transformations. Aussi peut-on suivre, depuis Proust jusqu'au Nouveau Roman, la désintégration d'une forme romanesque dépassée et la naissance d'une nouvelle esthétique capable de traduire l'univers nouveau dans lequel nous sommes entrés. Ce n'est donc pas un hasard si le personnage du roman traditionnel, avec nom, prénom, famille, hérédité, profession, champ de vigne ou compte en banque, a fait place à un être dont le visage ou même le nom demeurent souvent inconnus. Nous ne sommes plus au temps de Balzac où l'homme avait conscience d'exister en tant qu'individu, où il avait l'impression d'occuper le centre du monde. Comme Robbe-Grillet le fait remarquer dans son essai Pour un nouveau roman, les relations que nous entretenons avec l'univers ne sont plus des relations de possession: le monde demeure étranger, fermé sur lui-même, sinon hostile à l'homme. L'homme luimême tend à " se chosifier », pour employer une expression chère à Jean-Paul Sartre, il est en passe de devenir un étranger pour les autres et lui-même. On comprend dès lors la « nausée » du Roquentin de Sartre, son sentiment d'être « de trop ॥, on comprend aussi le comportement de Meursault, l'" étranger» de Camus; on n'est 
guère surpris de voir la prolifération des objets dans les romans de Robbe-Grillet ou de Butor, la dissection minutieuse et patiente de ce qui n'est même plus un état d'âme chez Sarraute. Est-ce à dire que l'homme a disparu pour faire place aux choses? Non, la présence de l'homme, si limitée soit-elle, est d'autant plus tragique que l'être humain se sent écrasé par la présence inhumaine d'objets en série. Les choses ne sont plus pour nous des "objets inanimés » qui ont une " âme ».

L'intrigue cohérente, rectiligne du roman balzacien ne peut plus rendre compte des nouvelles relations que I'homme noue avec le monde d'aujourd'hui. Si l'on en croit Robbe-Grillet, cette intrigue ordonnée, logique, avec sa " courbe régulière des passions, la tension de chaque épisode vers la fin ", correspondait à un ordre social stable et cohérent dont avait besoin la bourgeoisie pour maintenir son emprise sur le matériel. Le romancier ne se contente plus de savoir raconter; il ne cherche plus à expliquer le monde mais à le décrire: empruntant sa méthode à la phénoménologie, il décrit simplement la réalité, les phénomènes observés sans chercher à les rattacher à une cause quelconque, à un a priori métaphysique ou psychologique. Expliquant la démarche de la phénoménologie, Georges Gurvitch écrit ces lignes qui me semblent s'appliquer particulièrement bien à la méthode de plusieurs romanciers contemporains : il faut avant tout « ne chercher à rien expliquer et théoriser, mais seulement guider pour ( montrer > et retrouver les qualités originelles et irréductibles "; il importe en outre "de s'abstenir, pour commencer, de tout jugement sur l'existence, de renoncer au début à tout affirmation négative ou positive concernant le caractère vrai ou imaginaire des objets $"$.

Déchiffrement du monde et surtout réflexion sur notre façon de déchiffrer le monde, le roman, qui est entré dans cette «ère du soupçon " dont parle Nathalie Sarraute, exige beaucoup du lecteur. Celui-ci n'est plus un être passif qui se laisse charmer par un habile conteur ; il est de la partie, il invente en quelque sorte le roman tout autant que l'auteur. Ce que Butor disait dans son intervention au Colloque de Royaumont en 1959, on pourrait l'appliquer au lecteur: «II y a une certaine matière qui veut se dire; et en un sens ce n'est pas le romancier qui fait le roman, c'est le roman qui se fait tout seul, et le romancier n'est que l'instrument de sa mise au monde, son accoucheur [...] $)$.

Si l'étude dans sorı ensemble demeure intelligente, attentive à mettre en lumière la spécificité de chacune des œuvres replacées dans l'évolution du genre romanesque au $X X^{e}$ siècle, le lecteur aura l'impression que $\mathrm{M}^{\mathrm{me}}$ Zeltner regroupe parfois artificiellement les auteurs choisis. Ainsi, Robbe-Grillet, au lieu de prendre place aux côtés de Butor et Sarraute, se voit étudié dans le même chapitre que Queneau et Camus. L'auteur s'en rend fort bien compte, puisqu'elle écrit, p. 139: «On pourrait se demander s'il n'aurait pas été plus opportun de placer Robbe-Grillet aux côtés de Butor et de Nathalie Sarraute. Ses dernières œuvres révèlent, en effet, très nettement un développement vers l'intérieur. Toutefois, son art demeure centré sur l'existentialisme et il n'est guère possible de le détacher de cette perspective ". $J$ 'avoue ne pas voir très bien en quoi les romans de Sarraute et de Butor sont plus éloignés de l'existentialisme que ceux de Robbe-Grillet. Un tel classement risque de fausser les perspectives et de faire perdre de vue ce qu'il y a de commun dans la démarche de ces trois romanciers.

Le livre de $\mathrm{M}^{\mathrm{me}}$ Zeltner, qui puise abondamment chez les critiques allemands Kayser, Franzen et Rutsch, demeure à mi-chemin entre 
la vulgarisation et l'érudition; par sa profonde connaissance de la littérature européenne du $\mathrm{XX}^{\mathrm{e}}$ siècle, par sa largeur de vues et la subtilité de ses analyses, il constitue l'introduction la plus pénétrante au roman français des trente-cinq dernières années.

Université Laval

Réal OUELLET 